REVIEW ARTICLE

\author{
K.A. Tong \\ S. Ashwal \\ A. Obenaus \\ J.P. Nickerson \\ D. Kido \\ E.M. Haacke
}

\title{
Susceptibility-Weighted MR Imaging: A Review of Clinical Applications in Children
}

\begin{abstract}
SUMMARY: Susceptibility-weighted imaging (SWI) is a high-spatial-resolution 3D gradient-echo MR imaging technique with phase postprocessing that accentuates the paramagnetic properties of blood products such as deoxyhemoglobin, intracellular methemoglobin, and hemosiderin. It is particularly useful for detecting intravascular venous deoxygenated blood as well as extravascular blood products. It is also quite sensitive to the presence of other substances such as iron, some forms of calcification, and air. We have used this technique in the past several years to study a wide variety of pediatric neurologic disorders. We present a review with selected case histories to demonstrate its clinical usefulness in the improvement of the following: 1) detection of hemorrhagic lesions seen in various conditions, including traumatic brain injury and coagulopathic or other hemorrhagic disorders; 2) detection of vascular malformations such as cavernous angiomas, telangiectasias, or pial angiomas associated with Sturge-Weber syndrome; 3) demonstration of venous thrombosis and/or increased oxygen extraction in the setting of infarction, hypoxic/anoxic injury, or brain death; 4) delineation of neoplasms with hemorrhage, calcification, or increased vascularity; and 5) depiction of calcium or iron deposition in neurodegenerative disorders. SWI has provided new understanding of some of these disease processes. It is hoped that as SWI becomes more widely available, it will provide additional diagnostic and prognostic information that will improve the care and outcome of affected children.
\end{abstract}

$\mathbf{S}_{\mathrm{s}}^{\mathrm{u}}$ usceptibility-weighted imaging (SWI) is a high-spatialresolution 3D gradient-echo MR imaging technique with phase postprocessing that accentuates the paramagnetic properties of blood products and is very sensitive in the detection of intravascular venous deoxygenated blood as well as extravascular blood products. ${ }^{1}$ It was originally referred to as highresolution blood oxygen level-dependent venography, ${ }^{2-4}$ but because of its broader application than evaluating venous structures, it is now referred to as SWI. ${ }^{1,5}$

In the past decade, the clinical value of SWI in adults with neurologic disorders has been increasingly reported. ${ }^{5}$ SWI has been used in studies of arterial venous malformations, ${ }^{6}$ occult venous disease, ${ }^{4}$ multiple sclerosis, ${ }^{7}$ trauma, ${ }^{8,9}$ tumors, ${ }^{10-13}$ and functional brain imaging. ${ }^{14}$ SWI has been used at our institution since 2001, and in this review, we describe its role in providing additional imaging information in a wide variety of pediatric neurologic conditions.

\section{Principles of SWI}

SWI exploits the loss of signal intensity created by disturbance of a homogeneous magnetic field, the principles of which have been extensively described by Haacke et al, ${ }^{1}$ Reichenbach et al, ${ }^{2}$ and Sehgal et al. ${ }^{5}$ Briefly, these disturbances can be caused by various paramagnetic, ferromagnetic, or diamagnetic substances such as air/tissue or air/bone interfaces. As spins encounter heterogeneity in the local magnetic field, they precess at different rates and cause overall signal-intensity loss in $\mathrm{T} 2{ }^{\star}$ weighted (ie, gradient-echo) images. Sensitivity to susceptibil-

Received July 9, 2007; accepted July 13

From the Departments of Radiology (K.A.T., D.K.), Pediatrics (S.A.), and Radiation Medicine (A.0.), Loma Linda University School of Medicine, Loma Linda, Calif; Fletcher Allen Healthcare (J.P.N.), University of Vermont, Burlington, Vt; and Wayne State University (E.M.H.), Detroit, Mich.

Paper previously presented in part at: Annual Meeting of the American Society of Neuroradiology, Toronto, Ontario, Canada; April-May 2005.

Please address correspondence to Karen Tong, MD, Department of Radiology, Loma Linda University Medical Center, Loma Linda, CA 92354; e-mail: ktong@llu.edu

DOI 10.3174/ajnr.A0786 ity effects increase as one progresses from fast spin-echo to routine spin-echo to gradient-echo techniques, from T1- to T2- to $\mathrm{T}^{*}$-weighting, from short-to-long echo times, and from lower to higher field strengths.

Application of a magnetic field to the brain generates an induced field that depends on the applied magnetic field and on the magnetic susceptibility of molecules within the brain. Magnetic susceptibility variations are higher at the interface of 2 regions, and signal intensity changes are also dependent on other factors, including hematocrit, deoxyhemoglobin concentration, red blood cell integrity, clot structure, molecular diffusion, $\mathrm{pH}$, temperature, field strength, voxel size, previous contrast material use, blood flow, and vessel orientation. ${ }^{15}$

SWI is not a new concept, but recent advances have allowed the technique to be refined, thereby expanding its applicability to study various conditions and pathologic states. Haacke et $\mathrm{al}^{1}$ designed SWI, as first described in Reichenbach et al, ${ }^{2}$ because it is a high-spatial-resolution 3D fast low-angle shot (FLASH) MR imaging technique that is extremely sensitive to susceptibility changes. The underlying contrast mechanism is primarily associated with the magnetic susceptibility difference between oxygenated and deoxygenated hemoglobin, leading to a phase difference between regions containing deoxygenated blood and surrounding tissues, resulting in signalintensity cancellation. The term "SWI" implies full use of magnitude and phase information and is not simply a $\mathrm{T}^{*}$ imaging approach. A detailed explanation of the underlying physics and mathematic principles is beyond the scope of this clinical review, but several recent publications describe this information in depth. ${ }^{1,5}$

The original sequence consisted of a strongly susceptibility-weighted, low-bandwidth $(78 \mathrm{~Hz} /$ pixel $) 3 \mathrm{D}$ FLASH sequence $\left(\mathrm{TR} / \mathrm{TE}=57 / 40 \mathrm{~ms}\right.$, flip angle $\left.=20^{\circ}\right)$ first-order flowcompensated in all 3 orthogonal directions. Thirty-two to 64 partitions of $2 \mathrm{~mm}$ could be acquired by using a rectangular FOV (5/8 of $256 \mathrm{~mm})$ and a matrix size of $160 \times 512$, resulting in a voxel size of $1 \times 0.5 \times 2 \mathrm{~mm}^{3}$. The acquisition time varied from 5 to 10 minutes, depending on the desired coverage. 


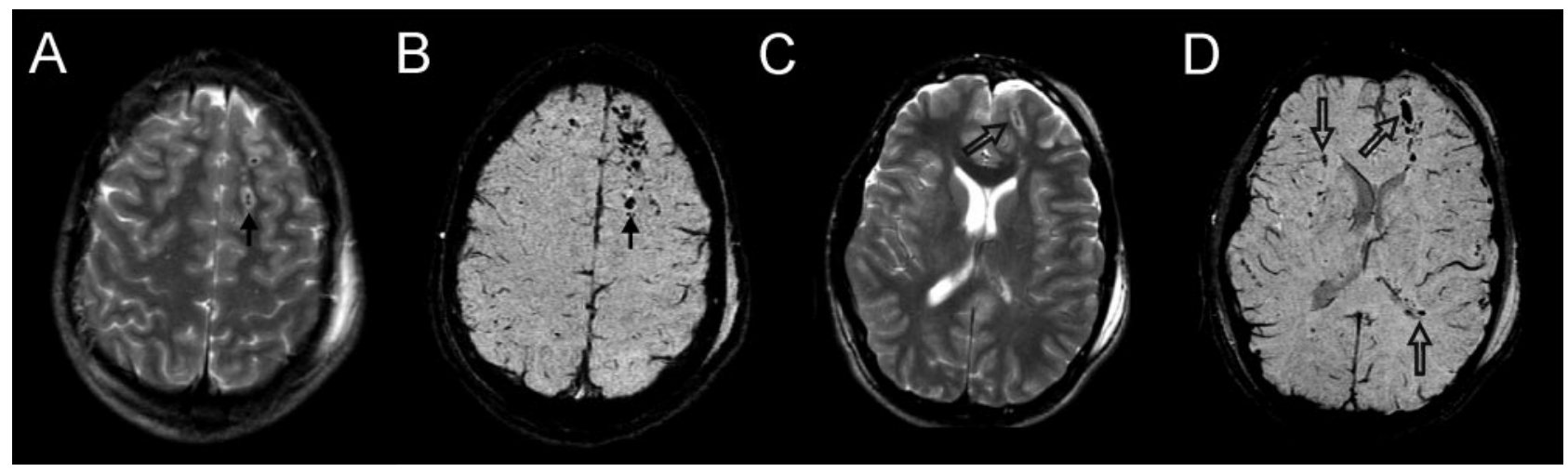

Fig 1. Diffuse axonal injury. This 17-year-old boy had a severe TBI in a motorcycle crash and had an initial GCS score of 5 . Intracranial pressure was normal. Axial T2 and SWI images at the level of the centrum semiovale $(A$ and $B)$ and at the level of the lateral ventricles $(C$ and $D)$ are depicted. Small hemorrhagic shearing injuries in the left frontal subcortical white matter (arrows) are more are visible on SWI (B). At the lower level, SWI (D) shows additional small hemorrhagic shearing foci (open arrows) in the left frontal white matter, right subinsular region, and left splenium that are only partly visible on T2-weighted images. He was discharged 16 days after admission with moderately impaired cognition. Two years after injury, he continues to have fine-motor deficits. He has significant impairments on measures of verbal functioning (verbal comprehension, learning, and memory).

After the data are acquired, there is additional postprocessing that accentuates the signal intensity loss caused by any susceptibility effects. This primarily involves the creation of a phase mask to enhance the phase differences between paramagnetic substances and surrounding tissue. It should be noted that there are enhancements of contrast just from the phase changes, even though there may be no $\mathrm{T} 2^{\star}$ effects and vice versa. ${ }^{1}$ There have been newer modifications to this sequence, including echo-planar techniques, to decrease the scanning time. The sequence, along with its automatic postprocessing, is currently available for Siemens (Erlangen, Germany) 1.5 and 3T MR imaging scanner platforms; currently SWI is being used in approximately 5-10 centers in the United States and several centers in Europe and Asia.

\section{Pediatric Clinical Applications of SWI}

As in adults, SWI has been found to be helpful in children with various disorders, including traumatic brain injury (TBI), coagulopathic or other hemorrhagic disorders, cerebral vascular malformations, venous shunt surgery or thrombosis, infarction, neoplasms, and degenerative/neurometabolic disorders associated with intracranial calcifications or iron deposition. The following sections discuss different pediatric clinical applications of SWI. Although phase information has been shown to be helpful in some adult conditions, this review will focus on the use of the routine postprocessed images.

\section{Detection of Hemorrhagic Lesions}

Hemorrhagic lesions are seen in a number of central nervous system conditions, including TBI, coagulopathies, and neoplasms. SWI has proved useful in increasing the sensitivity in detecting such hemorrhagic lesions, which can be helpful for management and prognosis.

TBI. Detection of large amounts of intracranial hemorrhage is of primary importance in the acute surgical management of patients with TBI. However, identification of smaller hemorrhages and their location now provides useful information regarding mechanism of injury and potential clinical outcome. This is particularly helpful for evaluation of diffuse axonal injury (DAI), which is often associated with small hemorrhages in the deep brain that are not routinely visible on CT or conventional MR imaging sequences. Most blood prod- ucts are paramagnetic (deoxyhemoglobin, intracellular methemoglobin, and hemosiderin), making it possible to exploit magnetic susceptibility effects. In acute and early subacute phases, these effects largely occur from deoxyhemoglobin and intracellular methemoglobin.

Several investigators have examined conventional gradient refocused echo (GRE) sequences in the setting of TBI. Scheid et $\mathrm{al}^{16}$ found that $\mathrm{T} 2^{\star}$-weighted GRE sequences detected significantly more lesions than conventional T1- or T2-weighted sequences in adult patients with TBI, by using high-field (3T) MR imaging. Although there was a correlation between the total amount of microhemorrhages and the Glasgow Coma Scale (GCS) score, they found no correlation with patient outcomes measured by the Glasgow Outcome Scale (GOS). Grados et $\mathrm{al}^{17}$ used a spoiled gradient-recalled T1-weighted MR imaging sequence to show that depth and number of lesions predicted outcome in children, though correlations were stronger with discharge outcomes compared with 1-year outcomes. Levin et $\mathrm{al}^{18}$ showed a correlation between depth of brain lesions (by using a combination of T1, T2, and GRE sequences) and outcomes measured by the GOS score as well as the Vineland Adaptive Behavior Scale-Revised. In all these studies, MR images were obtained in the chronic phase of TBI.

However, in a previous report, ${ }^{9}$ we showed, in 7 children with TBI (age, $14 \pm 4$ years; SWI, performed $5 \pm 3$ days after injury) that the number of hemorrhagic DAI lesions seen on SWI was 6 times greater than that on conventional T2*-weighted 2D GRE imaging and that the volume of hemorrhage was approximately twofold greater. As shown in Fig 1, SWI is particularly sensitive to the presence of very small hemorrhages. Although larger hemorrhages are visible on CT and conventional MR imaging sequences, numerous small hemorrhagic shearing lesions are only visible by using the SWI technique.

We have also used this technique to evaluate a larger group of 40 pediatric patients. ${ }^{8}$ We found that the extent of hemorrhage was strongly correlated with the initial severity of injury measured by the GCS score, the duration of coma, as well as the subsequent long-term outcome measured at 6-12 months after injury. We also found that most patients had lesions in frontal white matter or parieto-temporal-occipital gray or white matter. Several regions were less affected, including the thalamus, brain stem, cerebellum, and basal ganglia. There 


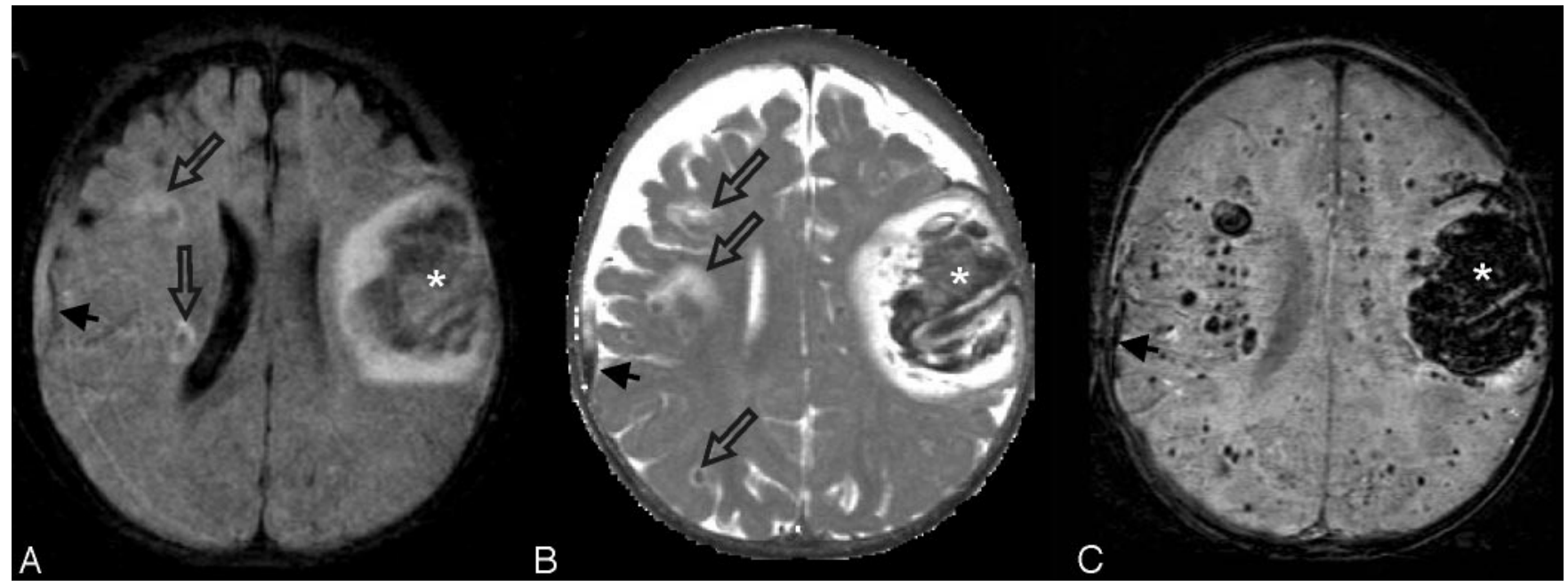

Fig 2. Leukemic hemorrhage. This 6-month-old boy presented with fever, leukocytosis thrombocytopenia, anemia, and hepatosplenomegaly. Axial FLAIR $(A)$, T2-weighted (B), and SWI ( $C$ images show a large hematoma in the left frontal lobe (asterisks). A few small hemorrhages with surrounding edema were also visible in the right subcortical white matter (open arrows) on the FLAIR and T2-weighted images. There is also a small right convexity subdural collection with hemorrhage (arrows). However, numerous additional hemorrhagic foci throughout the bilateral hemispheric white matter are only visible on SWI. He was subsequently diagnosed with acute lymphocytic leukemia. The multiple intracranial hemorrhages were thought to be associated with seizures that were difficult to control, with profound cognitive and motor impairments, and hydrocephalus that required ventriculoperitoneal shunting. Several years later he remains profoundly impaired.

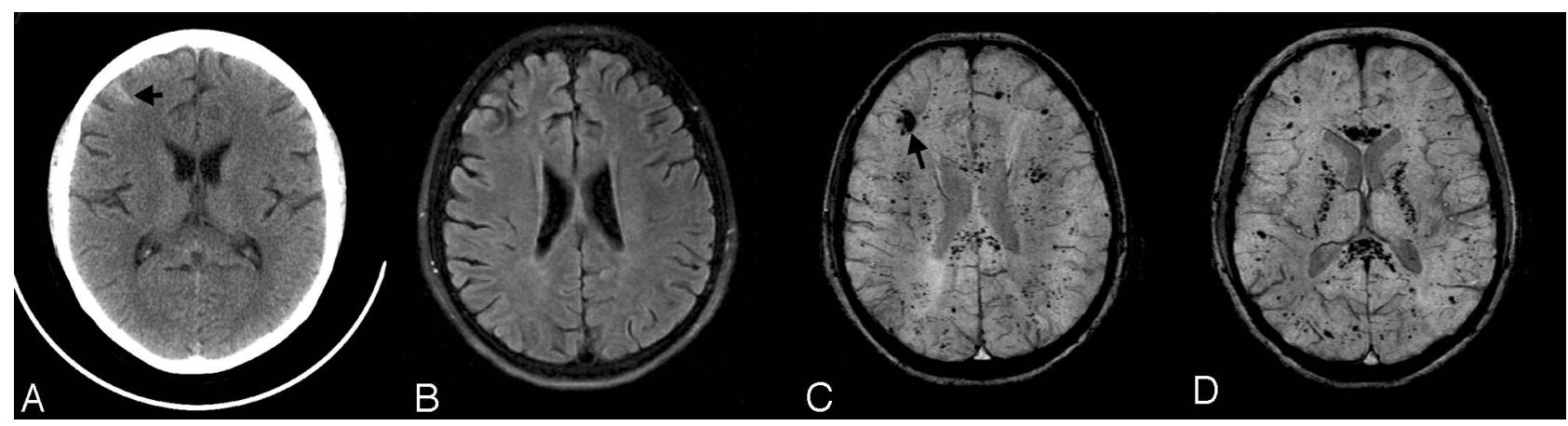

Fig 3. Vasculitis. This 16-year-old girl with systemic lupus erythematosus, chronic pancreatitis, nephritis, hypertension, cerebritis, and epilepsy presented with a 2-day history of chest pain and headache and a seizure on the day of admission. She was hypertensive and treated with methylprednisolone (Solu-Medrol) and cyclophosphamide (Cytoxan). Her complete blood count and chemistry profiles were normal, and she had no evidence of acute infection. Axial CT $(A)$, FLAIR $(B)$, and SWI $(C$ and $D)$ images are shown. A small hemorrhage is present in the right frontal lobe (arrows) on CT $(A)$ and SWI (C). However, only the SWI images demonstrate numerous additional tiny hypointense foci suggestive of petechial hemorrhages or possibly microthrombi throughout the white matter, particularly within the corpus callosum and internal capsules. She was placed on a higher dose steroid regimen and discharged 1 week after admission. Although the etiology of the lesions was undetermined, vasculitis or Cytoxan therapy were considered as possibilities.

were 14 patients who had lesions in 6 or fewer regions, and all had good outcomes at 6-12 months. Only patients with involvement of 7 or more regions were at risk for poor outcomes. More recently, we have shown that the number and volume of SWI hemorrhagic lesions correlated with specific neuropsychological deficits. ${ }^{19}$ These included measures of intelligence, executive skills, attention, visual-perceptual skills, language, verbal and nonverbal memory, motor functioning, and academic achievement (spelling, reading, and math). These findings suggest that the SWI technique can play an important role in more accurately diagnosing the degree of injury as well as providing valuable prognostic information that may help guide the management and rehabilitation of affected children. ${ }^{20}$ Considering the widespread consequences of DAI, its detection is important for the evaluation, treatment, and prognosis of patients with TBI, particularly those with moderate injuries in whom damage or repair may currently go unrecognized.

Other Types of Hemorrhagic Conditions. SWI is also useful in detecting other types of hemorrhagic lesions, such as those seen with coagulopathy, vasculitis, or some infections. In the setting of coagulopathy, either thrombosis (to be discussed later) or hemorrhage can occur, and the extent of bleeding may be underestimated. An example of this is shown in Fig 2, in which a 6-monthold infant presented with severe leukemia and had profound neurologic deficits and, even several years later, was profoundly incapacitated. Initially, it was uncertain as to why such severe deficits were present, but SWI revealed numerous extensive small hemorrhages throughout the brain that were not visible on other imaging sequences. In the setting of vasculitis, such hemorrhages may also go unnoticed without SWI, as shown in Fig 3. As in coagulopathic disorders, there may be a combination of microhemorrhage and microthrombi, which could potentially have a similar appearance. Pathologic correlation would be ideal but it is not often available. Follow-up imaging studies are also sometimes helpful.

\section{Vascular Malformations}

Certain types of vascular malformations with slow flow have been shown to be better visualized with SWI, including caver- 


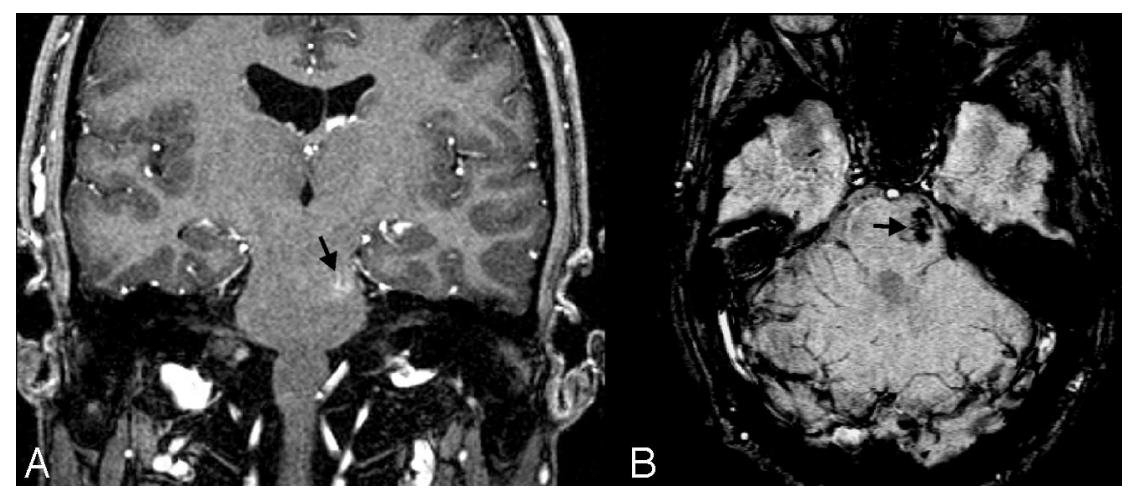

Fig 4. Cavernoma/telangiectasia. This 24-year-old student was being evaluated for partial complex seizures, and an MR image revealed a left temporal lobe lesion (not shown) that was thought to be a low-grade glioma, as well as an incidental lesion in the left pons. Contrast-enhanced coronal GRE T1-weighted image $(A)$ depicts a small mildly enhancing lesion in the left pons (arrow). The lesion was not visible on T2-weighted images (not shown) but appears on axial SWI $(B)$ as a markedly hypointense area. The ill-defined blush is commonly seen in low-flow vascular malformations, and the marked hypointensity of the lesion on the SWI sequence suggests that this represents an occult vascular malformation. Although there was no pathologic confirmation, given the location and size, it likely represents a cavernoma that had not bled.

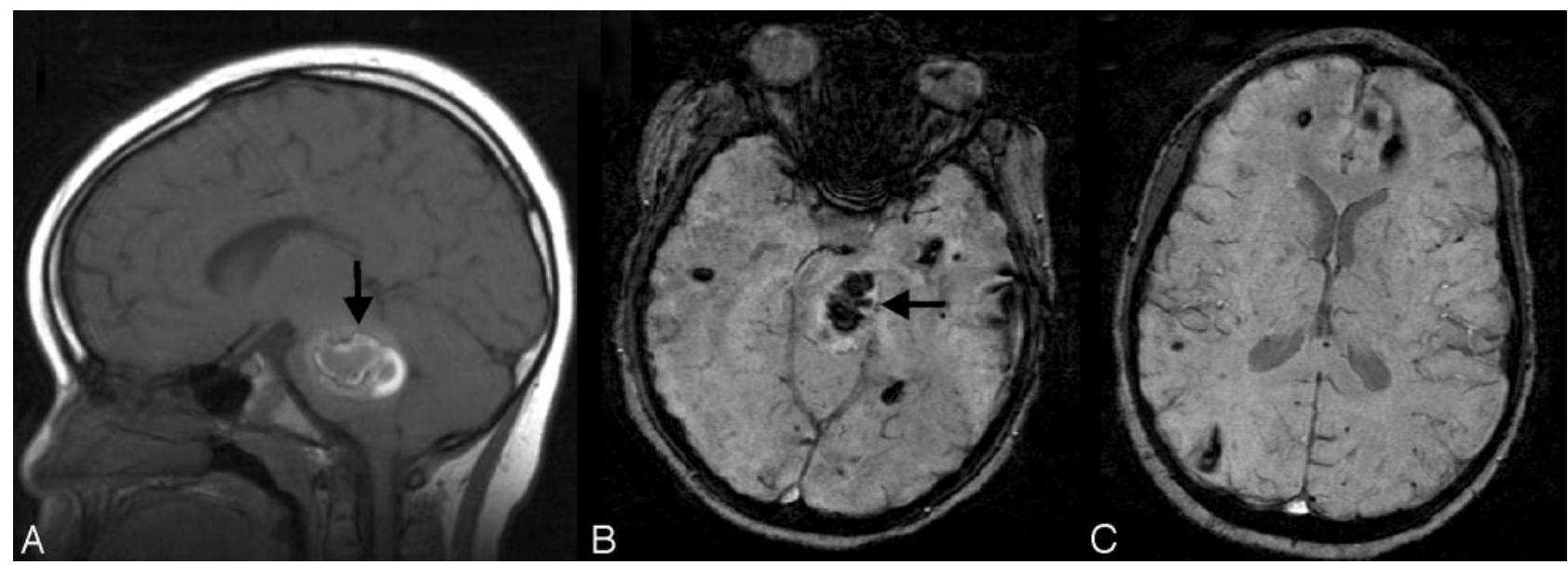

Fig 5. Multiple cavernous angiomas. This 7-year-old boy was admitted for evaluation of ataxia, dizziness, blurred vision, and alternating facial droop. MR imaging shows a large pontine hemorrhage (arrow) the on sagittal T1-weighted image $(A)$ and axial SWI $(B)$. Axial SWI at a higher level $(C)$ shows numerous additional scattered small hypointense lesions, consistent with multiple cavernomas. The pontine hemorrhage and cavernoma were subsequently surgically resected. He has been clinically stable.

nomas and telangiectasias. ${ }^{4,21}$ These types of low-flow malformations have been designated "occult" because they are usually not visible on conventional angiography, unlike high-flow arteriovenous malformations.

Cavernomas. Cavernomas that have previously bled are usually detectable on routine MR imaging due to the prominent signal intensity of hemorrhagic products. Cavernomas may also subsequently develop dystrophic calcifications that can be detected on CT. However, if these lesions are intact and have not bled, they may be almost invisible except for a faint or ill-defined blush of enhancement after contrast administration. However, contrast enhancement is inconsistent and often nonspecific. In addition, these lesions do not give flowrelated detectable signal intensity on conventional MR angiography techniques. SWI exploits the different relaxation rates between venous and arterial blood as well as the phase changes caused by the susceptibility differences between oxygenated and deoxygenated red blood cells. The technique is exquisitely sensitive to these small differences and enhances the signal-intensity loss in the venous circulation. As a result, these malformations become very dark on the SWI images, as shown in Fig 4. It should be noted that the effect is artifactually enhanced by this technique. The actual size of the lesion is likely smaller than the imaging abnormality-much like the "blooming" of hemorrhages seen with any GRE sequence. An example of SWI in a patient with multiple cavernomas is shown in Fig 5. In cases of ruptured cavernomas presenting as a first-time hemorrhage, the presence of multiple additional cavernomas is supportive evidence for diagnosis. Familial cavernomas are at especially high risk for hemorrhage and for developing new lesions. Cavernomas in the pons are common but hemorrhage can be devastating and these warrant close follow-up. It may be difficult to determine whether some small cavernomas are intact or have bled, unless there is additional supporting evidence on other sequences or serial imaging.

Telangiectasias. Telangiectasias are smaller and less common than cavernomas and can occur in mixed cavernoma/ telangiectasia lesions. These may occur sporadically or may be associated with syndromes (eg, hereditary hemorrhagic telangiectasia) or may occur as a result of endothelial injury, such as radiation-induced vascular injury, particularly in children who have received cranial irradiation. Although the occasional lesion is usually clinically benign, more extensive lesions may result in subtle neurologic symptoms for which no explanation may be found with routine imaging. However, SWI can detect these types of lesions, as shown in Fig 6 in this patient with previously diagnosed medulloblastoma, treated with radiation, who presented 8 years later with hearing loss and parieto-occipital headaches.

Sturge-Weber Syndrome. SWI is also helpful in the evaluation of children with vascular malformations and venous shunt surgery as seen in Sturge-Weber syndrome (SWS). SWS is thought to be a disorder of a primitive persistent plexus resulting in a pial angioma. This is associated with loss of normal cortical venous drainage, which could be congenital or resulting from progressive obliteration. The resulting abnor- 


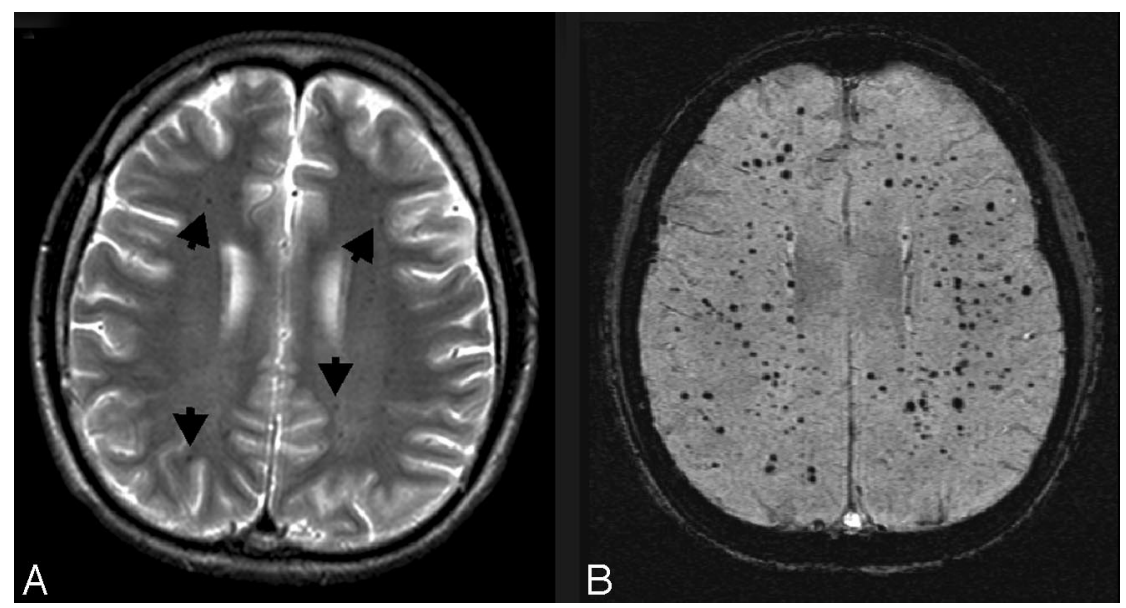

Fig 6. Radiation telangiectasias. This 11-year-old boy was diagnosed with medulloblastoma and had undergone surgical resection, radiation, and chemotherapy. He presented 8 years later with intermittent pulsating parieto-occipital headaches and complete loss of hearing in the left ear. Multiple serial MR imaging studies during the course of several years (not shown) showed a few scattered small faintly hypointense foci in the white matter but no other significant abnormalities. On his most recent MR imaging study, an axial T2-weighted image $(A)$ again shows a few small hypointense foci in the white matter (arrows), similar to those in prior studies. However, axial SWI $(B)$ shows numerous hypointense foci throughout the white matter, suggestive of radiation-induced telangiectasias.

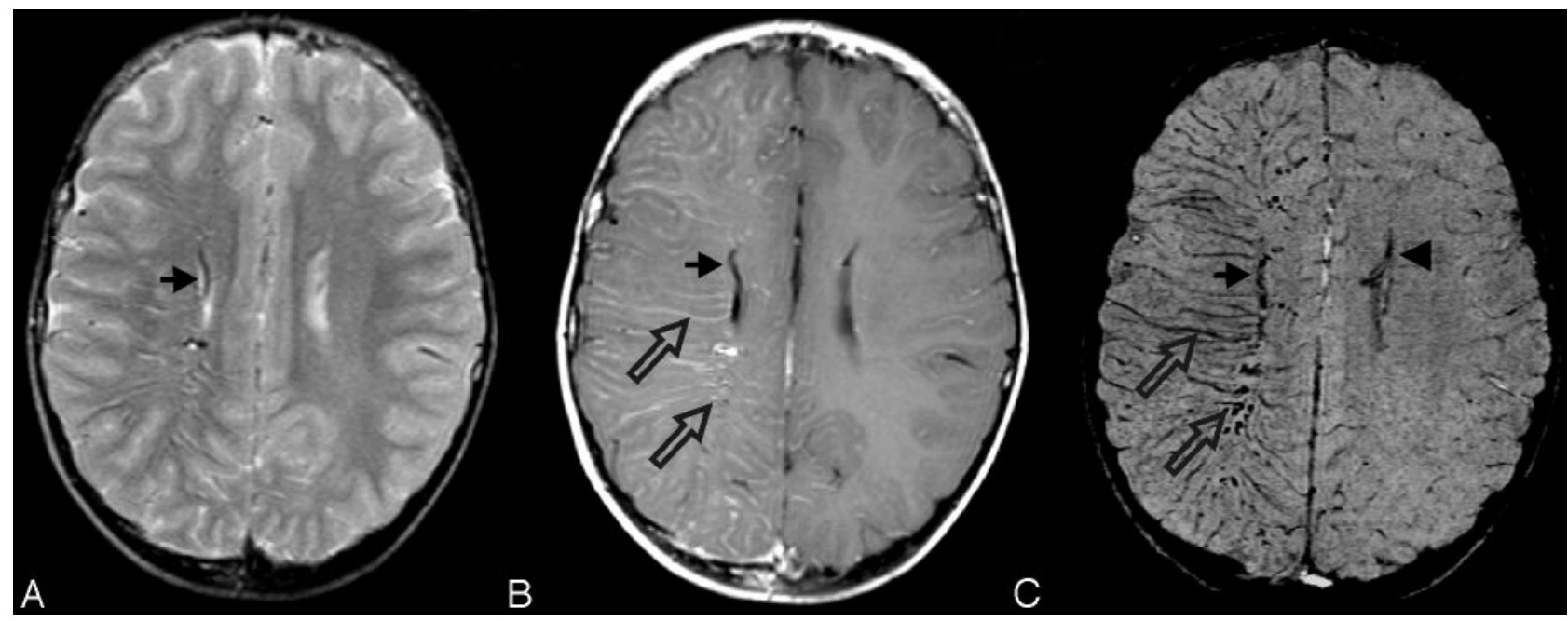

Fig 7. SWS. This 7-year-old boy was originally seen at 18 days of life and diagnosed with SWS on the basis of findings of bilateral port-wine stains involving the face, trunk, back, and thigh without evidence of hemihypertrophy. Findings of his electroencephalography were normal as were the findings of his neurologic examination. A CT scan in the neonatal period (not available) suggested prominent veins in the right meninges near the Sylvian fissure. His first MR imaging scan at 7 months (not shown) demonstrated intense contrast enhancement of the vascular structures of the right parietal and occipital regions. Follow-up MR imaging at age 5 years is shown. A mildly enlarged deep subependymal vein along the right lateral ventricle is visible (arrows) on a T2-weighted image $(A)$, contrast-enhanced T1-weighted image $(B)$, and SWI (C). The postcontrast T1-weighted image (B) also reveals additional prominently enhancing right subcortical and deep medullary veins (open arrows). However, SWI $(C)$ best delineates these prominent veins related to abnormal venous drainage in this condition and also shows a subtly prominent left subependymal vein (arrowhead), indicating early contralateral involvement. He has been developing normally, and his facial lesions have been treated with cosmetic laser surgery. He has had no evidence of seizures or other neurologic symptoms but is being treated for glaucoma.

mal venous drainage occurs through the deep venous system. Although this provides an initial compensatory mechanism that may portend a better prognosis, the deep venous circulation may be sluggish or associated with stasis, particularly if there is dural sinus stenosis or occlusion. The pial angioma is often easily seen with contrast-enhanced imaging. However, the deep veins are only occasionally visible, usually with conventional angiography. In typical patients with SWS, chronic venous ischemia eventually results in hemiatrophy and cortical "tram-track" calcification. Fishbein et $\mathrm{al}^{22}$ first described T2 shortening in the white matter of the affected hemisphere and suggested that this may be due to increased deoxyhemoglobin in the capillaries, from shunting of venous blood through the deep medullary veins. However, this speculation was based on conventional angiographic findings. Other investigators have suggested that the T2 shortening was due to abnormal myelination. We believe that we are now able to confirm Fishbein's explanation of white matter T2 shortening due to venous shunt surgery, as shown in Fig 7. The SWI technique remarkably demonstrates numerous deep medullary veins that are not well visualized by any other MR imaging sequence, particularly in the early mild cases of SWS. The increased visibility of veins is not only consistent with shunt surgery but may also imply increased oxygen extraction in the underlying brain parenchyma, particularly if there is associated deep venous stasis. This technique may also provide further understanding of the nature of tissue damage caused by a pial angioma. ${ }^{23}$

\section{Demonstration of Increased Veins Related to Venous Stasis or Increased Oxygen Extraction}

Venous Thrombosis. Sinovenous thromboses are increasingly being diagnosed in neonates, infants, and children, with the use of MR imaging and MR venography, and have contributed to the evaluation and management of such patients. An example of this occurs in patients with leukemia who develop a polyethyleneglycol (PEG)-asparaginase coagulopathy (Fig 8). SWI can be helpful in demonstrating unsuspected throm- 


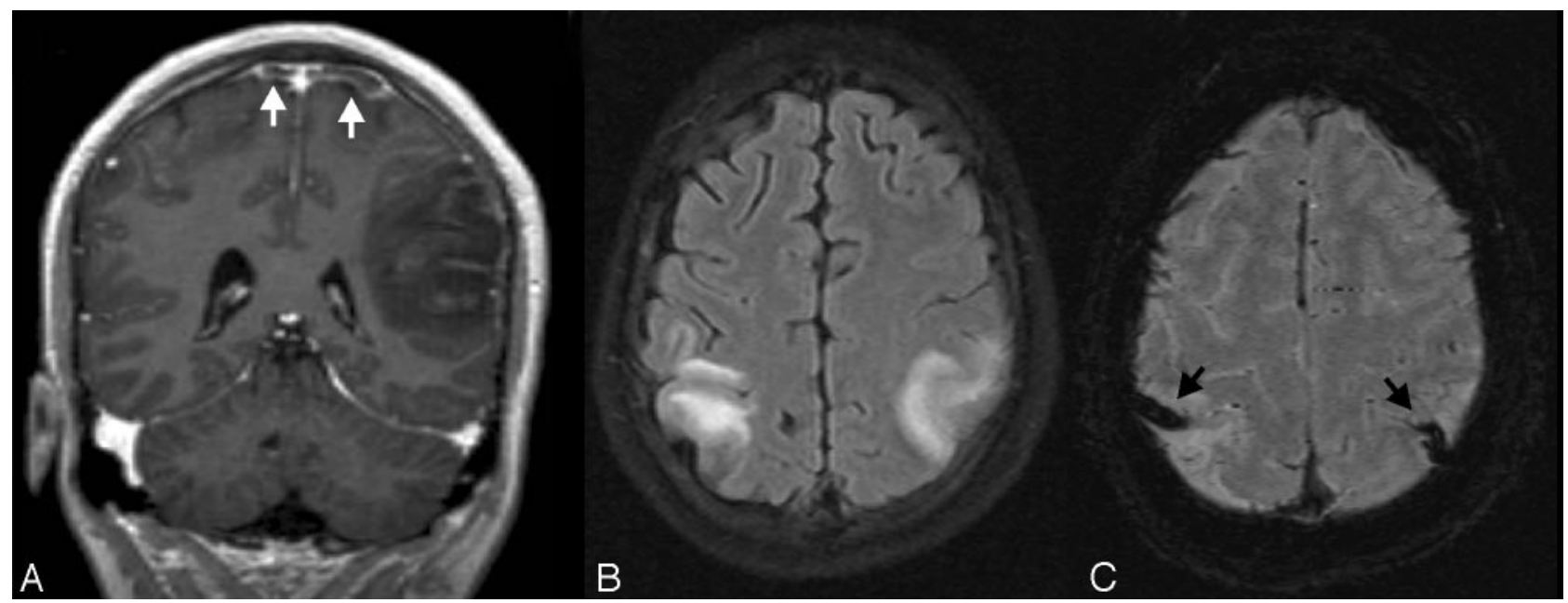

Fig 8. Cortical vein thrombosis. This 16-year-old boy with acute lymphoblastic leukemia presented with emesis, headache, and impaired speech. On the postcontrast coronal T1-GRE image $(A)$, there is a large irregular acute hemorrhage in the left parietal lobe with surrounding edema and adjacent sulcal effacement. Tubular filling defects are also visible on either side of the superior sagittal sinus (white arrows), consistent with thrombosed cortical veins. An axial FLAIR image (B) shows symmetric bilateral edema, adjacent to large hypointense cortical veins (black arrows) on SWI (C). In this case, the enlarged cortical veins were due to thrombosis, which was thought to be secondary to coagulopathy associated with PEG-asparaginase administration

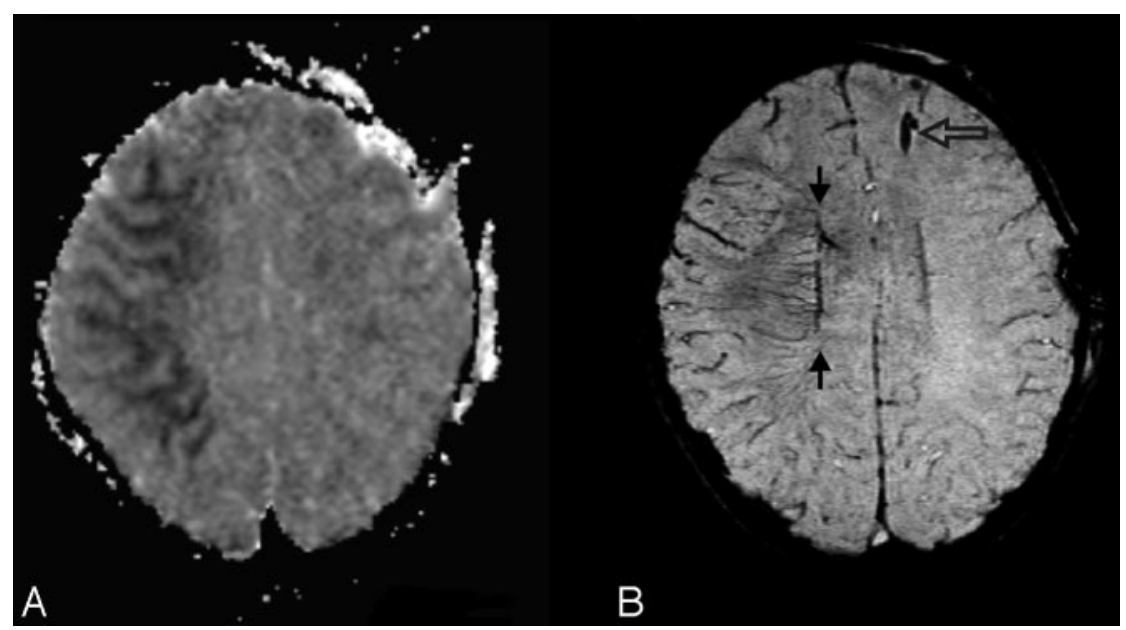

Fig 9. Prominent veins with infarct. This 3-year-old boy was ejected in a motor vehicle crash and was initially unresponsive with a GCS score of 3 . MR imaging 3 days after injury revealed diffuse axonal injury and contusions. Axial apparent diffusion coefficient map $(A)$ demonstrates dark signal intensity in the right middle cerebral artery territory, confirming a recent infarct. On SWI $(B)$, there are asymmetrically prominent deep medullary veins in the right hemisphere (arrow), suggestive of increased oxygen extraction. A ventriculostomy catheter is present in the left frontal region (open arrow). He was treated with $3 \%$ normal saline for increased intracranial pressure for 2 weeks, required mechanical ventilation for 3 weeks, and evolved from coma to a vegetative and then a minimally conscious state with severe bilateral spasticity without further improvement.

bosis to a much better degree of resolution than conventional imaging. Venous stasis likely results in greater prominence of the veins due to higher concentrations of intravascular deoxyhemoglobin. SWI can also demonstrate the extent of parenchymal brain hemorrhage that can occur after venous thrombosis that leads to infarction.

Focal Infarction. The possibility that SWI may also demonstrate increased oxygen extraction in areas of tissue infarction or hypoxemia has only recently been suggested, though not yet described in the literature, to our knowledge. We have seen several children who had increased deep medullary veins surrounding an area of infarction. We speculate that these veins reflect impaired cerebral blood flow in the penumbra around the infarct. In this zone, there should be lower levels of oxygenated red blood cells and, therefore, higher levels of deoxygenated red blood cells. This would account for the increased visibility of veins in these regions. In Fig 9, the strikingly asymmetric deep medullary veins just above the area of infarction are suggestive of increased oxygen extraction at the penumbra of the infarct. This might ultimately correlate with salvageable tissue at risk, though further investigation is necessary to confirm this.
Brain Death. Another example of prominent deep medullary veins has been seen in several cases of children with severe cerebral edema before brain death. As shown in Fig 10, SWI was helpful in this patient with TBI, who was sedated and paralyzed because the presence of diffusely prominent medullary veins suggested severe ischemic brain injury that was likely irreversible and predictive of brain death. The etiology of the increased visibility of deep veins remains uncertain but could reflect a combination of increased oxygen extraction, venous stasis, and/or possibly venous dilation secondary to release of substances such as adenosine $\mathrm{e}^{24}$ after cell death.

\section{Delineation of Neoplasms}

As in the adult population, ${ }^{10}$ SWI can increase the visibility of tumors and is helpful for depicting hemorrhage, calcification, and increased vascularity in some neoplasms, which may reflect tumor grade. Calcification in tumors might indicate slower growth and imply a lower grade, as seen in Fig 11. In rapidly growing tumors, hemorrhage into the tumor bed can initiate clinical presentation with headache, seizures, or progressive obtundation. Increased vascularity also suggests higher tumor grade, as seen in Fig 12, because neovascularity is 


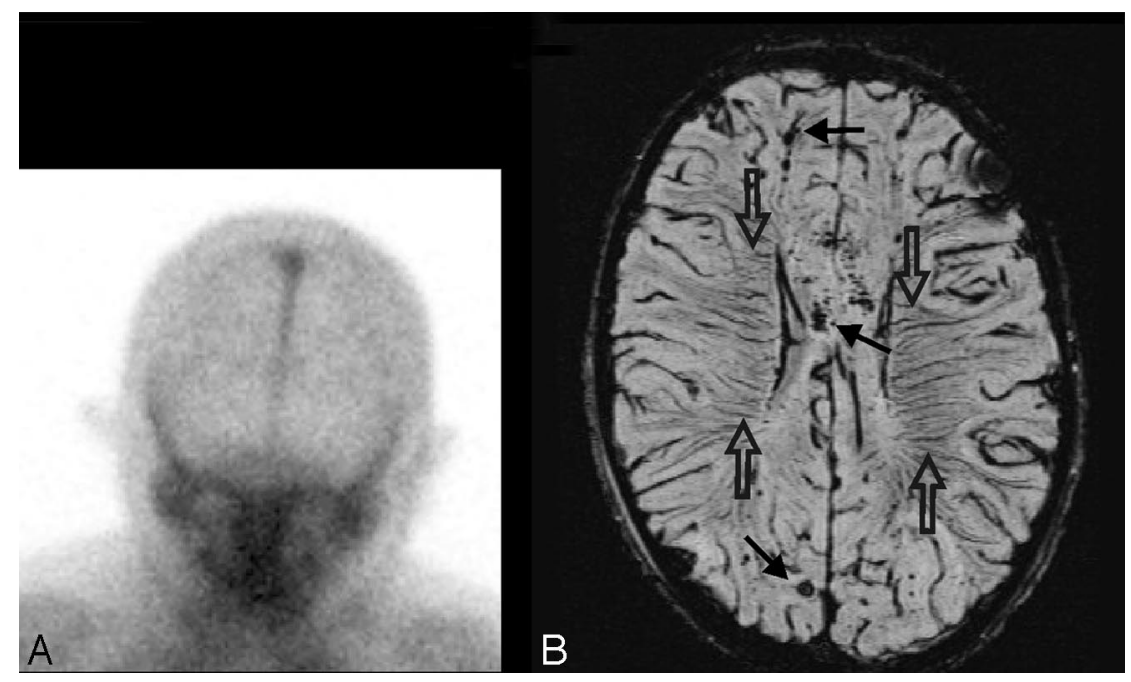

Fig 10. Prominent veins in brain death. This 8-year-old boy was ejected in a motor vehicle crash and sustained severe TBI with an initial GCS score of 3, nonreactive pupils, and markedly elevated intracranial pressure despite aggressive treatment. Anteroposterior view of nuclear medicine brain scan 5 days later $(A)$ demonstrates decreased but not absent cerebral blood flow as evidenced by activity in the superior sagittal sinus. MR imaging was obtained 11 days later. Axial SWI $(B)$ shows scattered small hypointense hemorrhages (arrows) in the subcortical white matter and corpus callosum, consistent with shearing injuries. In addition, there are prominent deep medullary veins (open arrows) throughout the bilateral hemispheres. He subsequently met clinical criteria for brain death and was taken off ventilatory support 2 weeks after admission.

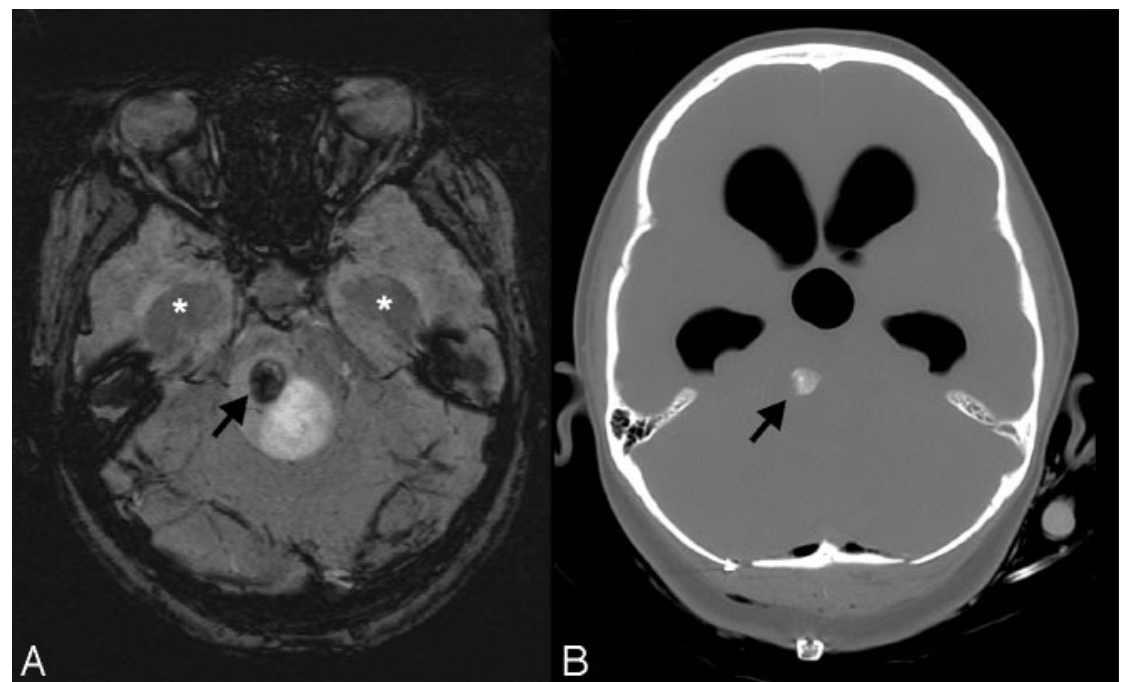

Fig 11. Calcification in tumor. This 12 -year-old girl had 2-month history of intermittent headaches, emesis, and left hemiparesis. Her initial CT scan (not available) showed moderate hydrocephalus and a fourth-ventricular mass. Conventional MR imaging images (not shown) demonstrated a partly cystic and partly solid enhancing mass. A smaller nonenhancing heterogeneous component in the right dorsal pons is noted to have a round area of markedly hypointense signal intensity (arrow) on SWI $(A)$ that corresponds to coarse calcification (arrow) on the postbiopsy CT (B). Dilation of the temporal horns (asterisks) due to obstructive hydrocephalus is also observed. Pathology revealed a diffusely infiltrating low-grade (I-II) astrocytoma with pilocytic features. The dark signal intensity on SWI was due to calcification in this low-grade glioma.

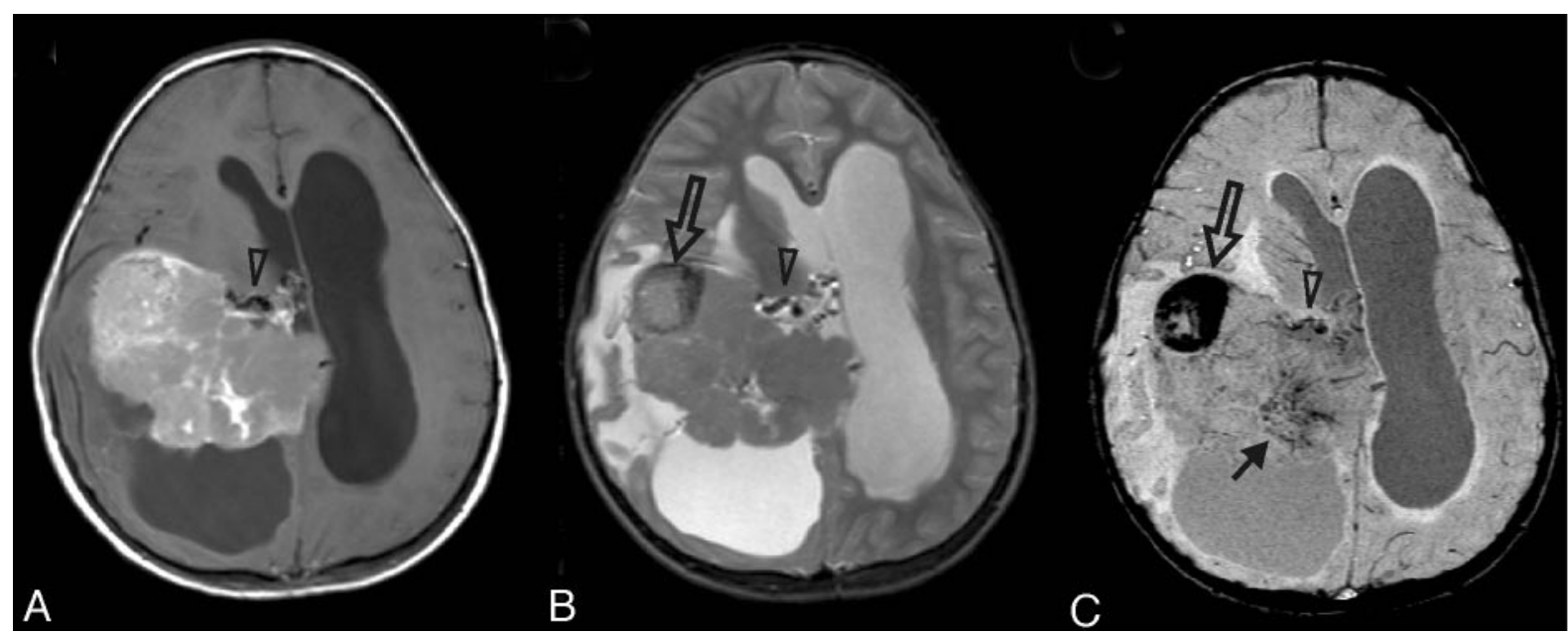

Fig 12. Increased vascularity in a tumor. This 8-year-old girl presented with headache, nausea, ataxia, and vomiting. CT imaging demonstrated a large mildly lobulated hyperattenuated mass arising from the right lateral ventricle with marked surrounding white matter edema and hydrocephalus. Axial postcontrast T1-weighted image $(A)$ shows patchy irregular enhancement of the lesion. The mass is largely isointense on the T2-weighted image $(B)$, except for a circular dark region (open arrow) that likely reflects an area of hemorrhage. On SWI $(C)$, the hemorrhage (open arrow) is markedly hypointense due to "blooming" effect. There are also small irregular hypointense areas in the posteromedial tumor, suggestive of increased venous vascularity (arrow). A mildly enlarged subependymal vein (arrowhead) is seen on all MR images. Pathology revealed a choroid plexus tumor with areas of anaplasia, including a high proliferative index, necrosis, and loss of architecture; most are consistent with choroid plexus carcinoma.

a reflection of tumor growth. Admittedly, it can sometimes be difficult to distinguish hemorrhage from abnormal venous vasculature. However, pre- and postcontrast SWI studies can potentially resolve this dilemma because blood vessels will 


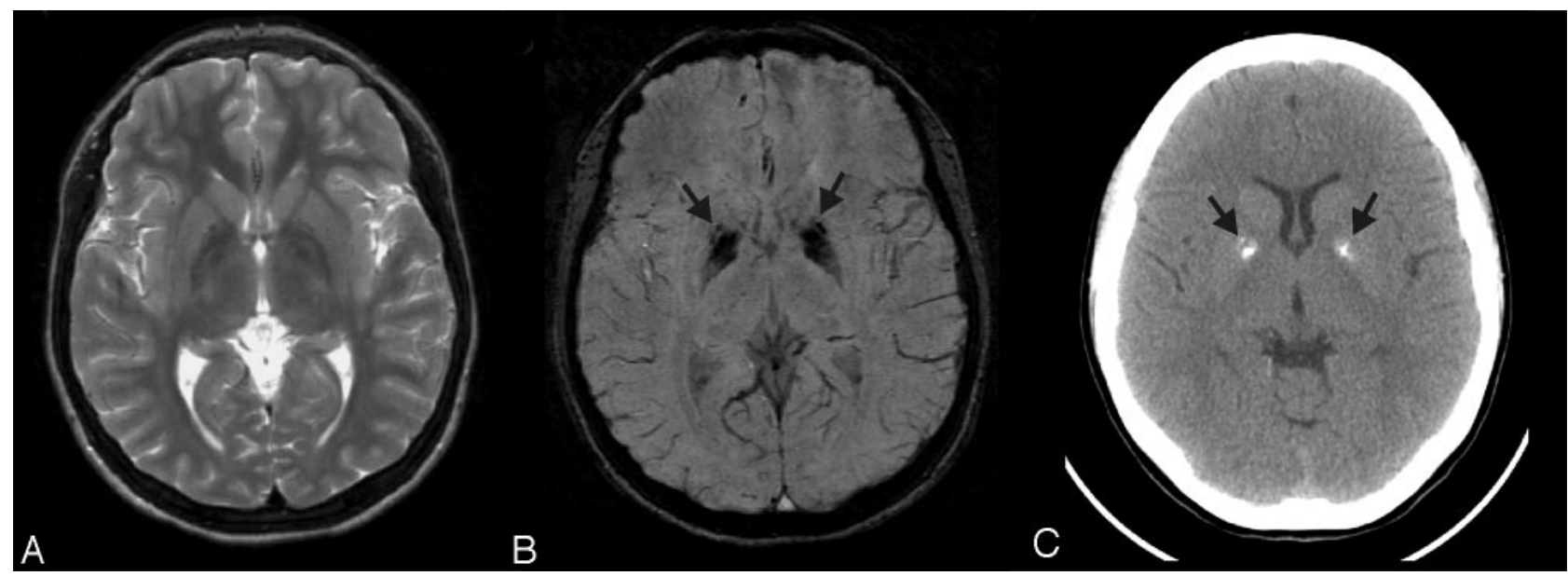

Fig 13. Fahr disease. This 15-year-old girl presented with tics, migraine headaches, and seizures. There is no significant abnormality on the T2-weighted $(A)$ image. However, SWI (B) reveals corresponding marked symmetric hypointensity in the anteromedial globus pallidi (arrows) that corresponds to irregular coarse calcification (arrows) on the CT image (C) - both of which are much greater than expected for her age. Without the SWI, the underlying disease might not be suspected. On the basis of the imaging findings and other affected family members, she was diagnosed as having Fahr disease (idiopathic basal ganglia calcification).

change in signal intensity after contrast, whereas areas of hemorrhage will not. SWI also has FLAIR-like contrast, whereupon CSF is suppressed but parenchymal edema remains bright ${ }^{5,10}$; which can also be helpful for delineation of tumor architecture. Information provided by SWI can better define the location of hemorrhage or neovascularity and may help guide the surgical or medical management of such patients.

\section{Depiction of Calcification or Iron Deposition}

Iron and calcium can be discriminated on the basis of their paramagnetic-versus-diamagnetic behaviors on the SWI filtered phase images. Generally, the veins will appear dark when parallel to the field (and when perpendicular depending on the resolution used) because the field increases. ${ }^{25}$ However, for small calcium deposits, the phase will appear brighter than the background because the field decreases. Usually, for small spheres, the dipole signature makes it quite clear whether the source is calcium or iron. ${ }^{26}$ Finally, if susceptibility mapping is used, it should be possible to collapse the phase information directly into a susceptibility map where dark signal intensity reflects iron and bright signal intensity reflects calcium. ${ }^{27}$

The utility of SWI in demonstrating iron or calcium deposition has been described in adult neurodegenerative disorders such as Parkinson and Alzheimer disease ${ }^{28}$ but can also be helpful in certain pediatric disorders. Calcium deposition can be visualized in certain genetic neurometabolic diseases such as Fahr disease (Fig 13), which is usually familial with autosomal dominant (variably expressive) inheritance. Calcium deposition is thought to be due to a disorder of neuronal calcium metabolism, associated with defective iron transport and free radicals that result in tissue damage and dystrophic calcification. Calcification typically occurs in the pallidal regions but can also involve the cerebellum and hemispheric white matter. Age at clinical presentation is variable with development of parkinsonian-like symptoms. Previously CT provided a better diagnostic specificity for cerebral calcification than MR imaging, but SWI may be more sensitive for early changes. Iron deposition can be seen in pantothenic kinase deficiency, which is a severe progressive neurodegenerative disorder. The etiology is not entirely understood, but the enzyme deficiencies are thought to be associated with energy and lipid dyshomeostasis, resulting in oxygen free radicals and phospholipid membrane destruction. The basal ganglia are thought be more susceptible to oxidative damage due to higher metabolic demand. The disease usually presents in childhood, with variable progression of neurologic deterioration. SWI allows better visualization of the extent of calcium and iron deposition, ${ }^{28}$ and in patients who present early in the disease course or in asymptomatic family members, the ability to detect early calcium deposition may be helpful for diagnosis, genetic counseling, and potential therapy once these become available.

\section{Conclusion}

We have shown that SWI can provide useful additional information in the evaluation of various pediatric neurologic conditions. Although not yet widely available, it is likely to become incorporated into the routine imaging assessment as improvements in software technology allow its acquisition and interpretation. As with many of the other recently introduced imaging techniques, initial studies in adults soon led to similar exploration in children, and in the case of SWI, its use in children should provide added diagnostic and therapeutic benefit.

\section{Acknowledgment}

The authors wish to thank Anna Smith for her technical help in preparation of the references.

\section{References}

1. Haacke EM, Xu Y, Cheng YC, et al. Susceptibility weighted imaging (SWI) Magn Reson Med 2004;52:612-18

2. Reichenbach JR, Venkatesan R, Schillinger DJ, et al. Small vessels in the human brain: $M R$ venography with deoxyhemoglobin as an intrinsic contrast agent. Radiology 1997;204:272-77

3. Cho ZH, Ro YM, Lim TH. NMR venography using the susceptibility effect produced by deoxyhemoglobin. Magn Reson Med 1992;28:25-38

4. Lee BC, Vo KD, Kido DK, et al. MR high-resolution blood oxygenation leveldependent venography of occult (low-flow) vascular lesions. AJNR Am J Neuroradiol 1999;20:1239-42

5. Sehgal V, Delproposto Z, Haacke EM, et al. Clinical applications of neuroimaging with susceptibility-weighted imaging. J Magn Reson Imaging 2005;22:439-50 
6. Essig M, Reichenbach JR, Schad L, et al. High resolution MR-venography of cerebral arteriovenous malformations [in German]. Radiologe 2001;41:288-95

7. Tan IL, van Schijndel RA, Pouwels PJ, et al. MR venography of multiple sclerosis. AJNR Am J Neuroradiol 2000;21:1039-42

8. Tong KA, Ashwal S, Holshouser BA, et al. Diffuse axonal injury in children: clinical correlation with hemorrhagic lesions. Ann Neurol 2004;56:36-50

9. Tong KA, Ashwal S, Holshouser BA, et al. Hemorrhagic shearing lesions in children and adolescents with posttraumatic diffuse axonal injury: improved detection and initial results. Radiology 2003;227:332-39

10. Sehgal V, Delproposto Z, Haddar D, et al. Susceptibility-weighted imaging to visualize blood products and improve tumor contrast in the study of brain masses. J Magn Reson Imaging 2006;24:41-51

11. Reichenbach JR, Jonetz-Mentzel L, Fitzek C, et al. High-resolution blood oxygen-level dependent MR venography (HRBV): a new technique. Neuroradiology 2001;43:364-69

12. Schad LR. Improved target volume characterization in stereotactic treatment planning of brain lesions by using high-resolution BOLD MR-venography. NMR Biomed 2001;14:478-83

13. Barth M, Nobauer-Huhmann IM, Reichenbach JR, et al. High-resolution three-dimensional contrast-enhanced blood oxygenation level-dependent magnetic resonance venography of brain tumors at 3 Tesla: first clinical experience and comparison with 1.5 Tesla. Invest Radiol 2003;38:409-14

14. Reichenbach JR, Haacke EM. High-resolution BOLD venographic imaging: a window into brain function. NMR Biomed 2001;14:453-67

15. Hermier M, Nighoghossian N. Contribution of susceptibility-weighted imaging to acute stroke assessment. Stroke 2004;35:1989-94

16. Scheid R, Preul C, Gruber O, et al. Diffuse axonal injury associated with chronic traumatic brain injury: evidence from $\mathrm{T} 2{ }^{*}$-weighted gradient-echo imaging at 3T. AJNR Am J Neuroradiol 2003;24:1049-56

17. Grados MA, Slomine BS, Gerring JP, et al. Depth of lesion model in children and adolescents with moderate to severe traumatic brain injury: use of SPGR
MRI to predict severity and outcome. I Neurol Neurosurg Psychiatry 2001;70:350-58

18. Levin HS, Mendelsohn D, Lilly MA, et al. Magnetic resonance imaging in relation to functional outcome of pediatric closed head injury: a test of the Ommaya-Gennarelli model. Neurosurgery 1997;40:432-40

19. Babikian T, Freier MC, Tong KA, et al. Susceptibility weighted imaging: neuropsychologic outcome and pediatric head injury. Pediatr Neurol 2005;33:184-94

20. Ashwal S, Holshouser BA, Tong KA. Use of advanced neuroimaging techniques in the evaluation of pediatric traumatic brain injury. Dev Neurosci 2006;28:309-26

21. Lee DH. MRI techniques: bilateral findings and "normal findings." $\mathrm{CanJNeu-}$ rol Sci 2000;27(suppl 1):S35-S38

22. Fischbein NJ, Barkovich AJ, Wu Y, et al. Sturge-Weber syndrome with no leptomeningeal enhancement on MRI. Neuroradiology 1998;40:177-80

23. Juhasz C, Haacke EM, Hu J, et al. Multimodality imaging of cortical and white matter abnormalities in Sturge-Weber syndrome. AJNR Am J Neuroradiol 2007;28:900-06

24. Bell MJ, Robertson CS, Kochanek PM, et al. Interstitial brain adenosine and xanthine increase during jugular venous oxygen desaturations in humans after traumatic brain injury. Crit Care Med 2001;29:399-404

25. $\mathrm{Xu} \mathrm{Y,} \mathrm{Haacke} \mathrm{EM.} \mathrm{The} \mathrm{role} \mathrm{of} \mathrm{voxel} \mathrm{aspect} \mathrm{ratio} \mathrm{in} \mathrm{determining} \mathrm{apparent}$ vascular phase behavior in susceptibility weighted imaging. Magn Reson Imaging 2006;24:155-60

26. Deistung A, Mentzel HJ, Rauscher A, et al. Demonstration of paramagnetic and diamagnetic cerebral lesions by using susceptibility weighted phase imaging (SWI). Z Med Phys 2006;16:261-67

27. Neelavalli J, Chen YCN, Haacke EM. Improved Fourier based Method for Calculating Field Inhomogeneity from Known Susceptibility Distribution: Proceeding of the International Society of Magnetic Resonance in Medicine, May 19-25, 2007.15:1016

28. Haacke EM, Cheng NY, House MJ, et al. Imaging iron stores in the brain using magnetic resonance imaging. Magn Reson Imaging 2005;23:1-25 